\title{
Analytical Study of Diagnosis for Angioplasty and Stents Patients using Improved Classification Technique
}

\author{
Faisal Riyaz Wani ${ }^{1}$, Er. Harwinder Kaur ${ }^{2}$ \\ ${ }^{1}$ M.Tech CSE, ${ }^{2}$ Assistant Professor \\ Department of Computer Sc. \& Engineering, St. Soldier Institute of Engineering \& Technology, \\ Near NIT, Jalandhar, Punjab, India
}

\begin{abstract}
The various technologies of data mining (DM) models for forecast of heart disease are discussed. Data mining plays an important role in building an intelligent model for medical systems to detect heart disease (HD) using data sets of Angioplasty and Stents patients, which involves risk factor associated with heart disease. Medical practitioners can help the patients by predicting the heart disease before occurring. The large data available from medical diagnosis is analyzed by using data mining tools and useful information known as knowledge is extracted. Mining is a method of exploring massive sets of data to take out patterns which are hidden and previously unknown relationships and knowledge detection to help the better understanding of medical data to prevent heart disease. There are many DM techniques available namely Classification techniques involving Naïve bayes (NB), Decision tree (DT), Neural network (NN), Genetic algorithm (GA), Artificial intelligence (AI) and Clustering algorithms like KNN, and Support vector machine (SVM). Several studies have been carried out for developing prediction model using individual technique and also by combining two or more techniques. This research provides a quick and easy review and understanding of available prediction models using data mining. The comparison shows the accuracy level of each model given by different researchers.
\end{abstract}

Key Words: DM, ICA, OneR, ZeroR

TRENDS IN USING DATAMINING TECHNIQUES IN HEART DISEASE

Although applying data mining is beneficial to healthcare, disease diagnosis, and treatment, few researches have investigated producing treatment plans for patients. Accurate diagnosis and treatment given to patients have been major issues highlighted in medical services. Recently, researchers started investigating using data mining techniques to handle the error and complexity of treatment processes for healthcare providers. Razali and Ali investigated generating treatment plans for acute upper respiratory infection disease patients using a decision tree. The model recommended treatment through giving drugs to patients showing accuracy of $94.73 \%$. Applying association rules and decision tree to treatment plans are showing acceptable performance. However, the comparison with other data mining techniques such as naîve bayes, neural network, and genetic algorithms still needs investigation.

\section{DATA MINING IN PREDICTION OF HEART DISEASE}

Despite the fact that data mining has been around for more than one decade, its potential is just been realized now. Data mining join factual examination, machine learning and database engineering to extract hidden patterns and connections from substantial databases Data mining mainly uses two methodologies:

supervised and unsupervised learning's. In supervised learning, a training set is utilized to learn model parameters though in unsupervised learning no training set is utilized like in K-Means clustering. The two most normal modeling goals of data mining are classification and prediction. Classification models classify discrete unordered values or data whereas prediction models predicts about continuous valued. Decision trees and Neural Networks are examples of classification models while Regression, Association 
Rules and Clustering are examples of prediction algorithm.

In this prediction of heart disease, we will use the following classification models of data mining are analyzed:

A. Decision trees

B. Neural networks

C. Naive Bayes Classifier

\section{PROPOSED SYSTEM}

To enhance the prediction of classifiers, genetic search is integrated; the genetic search resulted in 13 attributes which contributes more towards the prediction of the cardiac disease. The classifiers ensemble algorithms such as Random Forest are used for prediction of patients with heart disease. The classifiers are fed with reduced data set with 13 attributes. Results are shown in. Observations exhibit that the Proposed classification technique outperforms other data mining techniques such as Naïve Bayes and Decision Tree after incorporating feature subset selection but with high model construction time. Proposed classification technique performs consistently before and after reduction of attributes with the same model construction time.

The proposed system will have the following features:

$>$ Collection of health care data of heart disease.

$>$ Storage and Processing of data using Weka

$>$ Applying Proposed classification algorithm.

$>$ Analyzing performance in terms of accuracy and error rate.

\section{BJECTIVES OF THE PROPOSED STUDY}

The purpose of this research work to increase the efficiency of improved classification technique. The main purpose of this goal is to increase the efficiency and accuracy of the decision tree dataset.
1. To study the attribute of angioplasty and stent patients from various hospitals.

2. To refine the dataset for improving accuracy, correctly classified instance and reduce error rate.

3. To apply traditional and proposed algorithm on mixed dataset.

4. To analyze the computation parameters for improving efficiency of data set.

5. To critically analyze the diagnosis for angioplasty and stents patients using a improved classification technique.

6. To suggest self-programmed algorithm for improving efficiency of decision tree technique.

This research work focuses on above stated objectives which aim in increase the efficiency on correctly classified instances and reducing error rate.

\section{Improved Classification Algorithm}

The Improved Classification Algorithm is explained as below:

Input: Dataset

Output: Predicted class label

set number of classes to $N$, number of features to $M$

set $m$ determine the number of features at a node of decision

tree, $(m<M)$

for each decision tree do

Select randomly, a subset (with replacement) of training data that represents the $N$ classes and use the rest of data to measure the error of the tree

for each node of this tree do

Select randomly: $m$ features to determine the decision at the

node and calculate the best split accordingly.

end for

end for

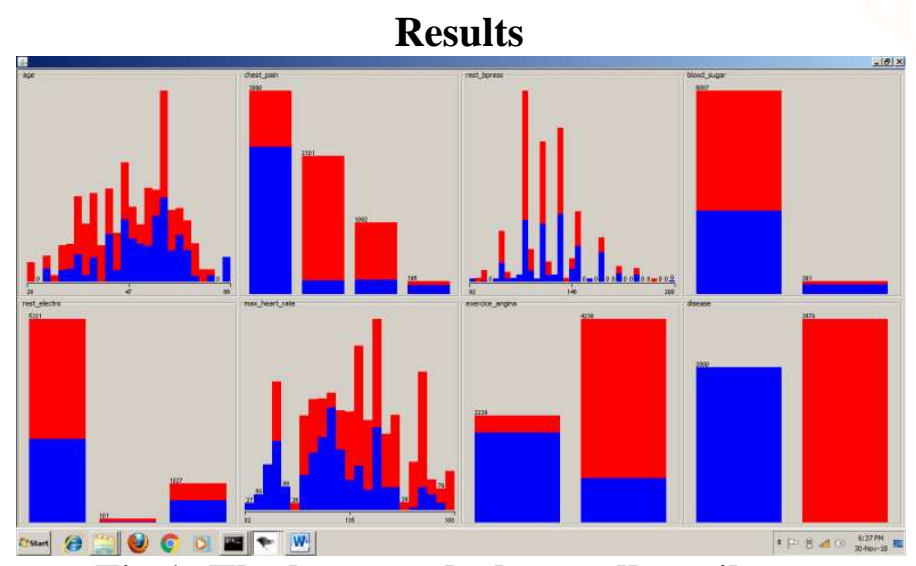

Fig 1: The bar graph shows all attributes 


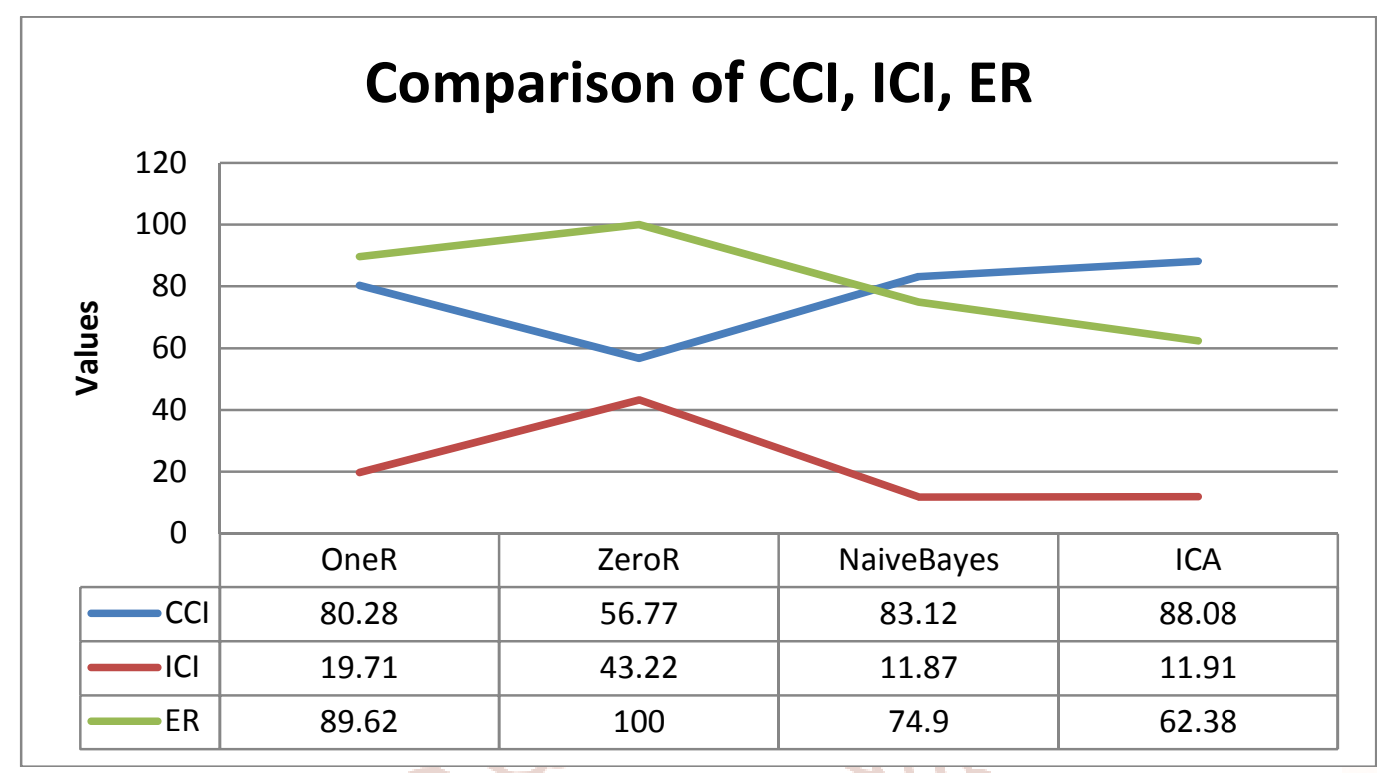

Fig 2 Shows the values of CCI, ICI and Error Rate of OneR, ZeroR, Naïve Bayes, and ICA Algorithms

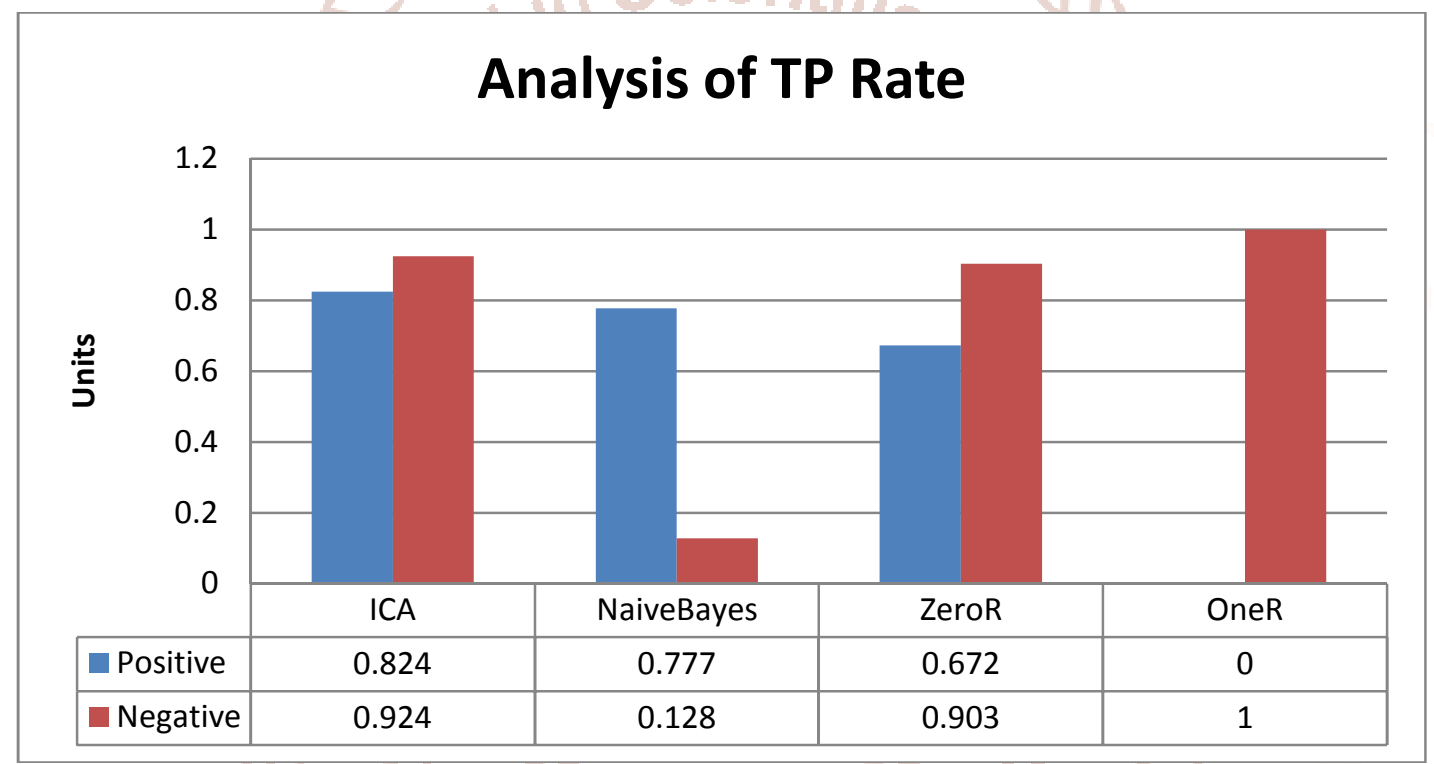

Fig 3: Shows the values of TP Rate of OneR, ZeroR, Naïve Bayes, and ICA Algorithms

\section{CONCLUSION}

Motivated by the world-wide increasing mortality of heart disease patients each year and the availability of huge amounts of data, researchers are using data mining techniques in the diagnosis of heart disease. Although applying data mining techniques to help health care professionals in the diagnosis of heart disease is having some success, the use of data mining techniques to identify a suitable treatment for heart disease patients has received less attention. Also, applying hybrid data mining techniques has shown promising results in the diagnosis of heart disease, so applying improved data mining technique in selecting the suitable treatment for heart disease patients needs further investigation. This research identifies gaps in the research on heart disease diagnosis and treatment and proposes a model to systematically close those gaps to discover if applying data mining techniques to heart disease treatment data can provide as reliable performance as that achieved in diagnosing heart disease patients.

Improved Classification Algorithm (ICA) shows the better results in correctly classified instances, incorrect classified instances, error rate, TP Rate, FP Rate, Precision, Recall and F-Measure Values.

\section{REFERENCES}

1. Aakash Chauhan, Aditya Jain, Purushottam Sharma, "Heart Disease Prediction using Evolutionary Rule Learning", International Conference on "Computational Intelligence and Communication Technology" (CICT 2018). 
2. M. Deepika, Dr. K. Kalaiselvi, “A Empirical study on Disease Diagnosis using Data Mining Techniques", Proceedings of the 2nd International Conference on Inventive Communication and Computational Technologies (ICICCT 2018) IEEE Xplore Compliant - Part Number: CFP18BAC-ART; ISBN:978-1-5386-1974-2.

3. Sarath Babu, Vivek EM, Famina KP, Fida K, Aswathi P, Shanid M, Hena M, "Heart Disease Diagnosis Using Data Mining Technique", International Conference on Electronics, Communication and Aerospace Technology ICECA 2017.

4. Salma Banu N.K, Suma Swamy, "Prediction of Heart Disease at early stage using Data Mining and Big Data Analytics: A Survey", International Conference on Electrical, Electronics, Communication, Computer and Optimization Techniques (ICEECCOT), 2016.

5. Ankita Dewan, Meghna Sharma, "Prediction of Heart Disease Using a Hybrid Technique in Data Mining Classification", 2nd International Conference on Computing for Sustainable Global Development (INDIACom), 2015.

6. Monika Gandhi, Dr. Shailendra Narayan Singh, "Predictions in Heart Disease Using Techniques of Data Mining",1st International Conference on Futuristic trend in Computational Analysis and Knowledge Management (ABLAZE-2015).

7. M. A. JABBAR, B.L Deekshatulu, Priti Chndra, "Alternating decision trees for early diagnosis of heart disease", Proceedings of International Conference on Circuits, Communication, Control and Computing (I4C 2014).

8. M. A. Jabbar, Shirina samreen, "Heart disease prediction system based on hidden naïve bayes classifier", GJCST, Volume 13 Issue 3, Ver 1.0, 15-25,(2013).

9. Shubpreet Kaur, Dr. R.K.Bawa, "Implementation of an Expert System for the Identification of Drug Addiction Using Decision Tree ID3 Algorithm", IEEE, 2017.

10. Priyanka N, Dr.Pushpa RaviKumar," Usage of Data mining techniques in predicting the Heart diseases - Naïve Bayes \& Decision tree", International Conference on circuits Power and Computing Technologies [ICCPCT], 2017.

11. Purushottam, Prof. (Dr.) Kanak Saxena, Richa Sharma," Efficient Heart Disease Prediction
System using Decision Tree", International Conference on Computing, Communication and Automation (ICCCA2015).

12. Rashmi G Saboji, Prem Kumar Ramesh," A Scalable Solution for Heart Disease Prediction using Classification Mining Technique”, International Conference on Energy, Communication, Data Analytics and Soft Computing (ICECDS-2017).

13. Mai Shouman, Tim Turner, Rob Stocker, "USING DATA MINING TECHNIQUES IN HEART DISEASE DIAGNOSIS AND TREATMENT", Japan-Egypt Conference on Electronics, Communications and Computers,2012.

14. C. Sowmiya, Dr. P. Sumitra, "Analytical Study of Heart Disease Diagnosis Using Classification Techniques", IEEE INTERNATIONAL CONFERENCE ON INTELLIGENT TECHNIQUES IN CONTROL, OPTIMIZATION AND SIGNAL PROCESSING, 2017.

15. P. Sudeshna, S. Bhanumathi, M. R, Anish Hamlin," Identifying Symptoms and Treatment for Heart Disease from Biomedical Literature Using = Text Data Mining", International Conference on Computation of Power, Energy, Information and Communication (ICCPEIC), 2017.

16. Theresa Princy. R, J. Thomas, "Human Heart Disease Prediction System using Data Mining Techniques", International Conference on Circuit, Power and Computing Technologies [ICCPCT], 2016.

17. Yong Wang, Ya-Wei Zhao," Transplantation of Data Mining Algorithms to Cloud Computing Platform when Dealing Big Data", International Conference on Cyber-Enabled Distributed Computing and Knowledge Discovery, 2014.

18. Huihui Zhao, Jianxin Chen, Qin Hou, "Characteristic Pattern Study of Coronary heart disease with Qi-yin deficiency syndrome based on Decision Tree", Second International Conference on Intelligent Computation Technology and Automation, 2009.

19. Huihui Zhao, Shuwen Guo, Jianxin Chen , Qi Shi, Juan Wang, Chenglong Zheng, Peng Tan," Characteristic Pattern Study of Coronary Heart Disease with Blood Stasis Syndrome Based on Decision Tree", of biological systems, vol. 15, Dec. 2013, pp. 435-451. 